Théologiques

Théologiques

\title{
Empathie et expérience humaine
}

Essai de circulation mutuelle entre science cognitive, sagesse contemplative et philosophie phénoménologique

\section{Evan Thompson}

Volume 12, numéro 1-2, 2004

Le Soi dans tous ses états

URI : https://id.erudit.org/iderudit/011555ar

DOI : https://doi.org/10.7202/011555ar

Aller au sommaire du numéro

\section{Éditeur(s)}

Faculté de théologie et de sciences des religions, Université de Montréal

\section{ISSN}

1188-7109 (imprimé)

1492-1413 (numérique)

Découvrir la revue

Citer cet article

Thompson, E. (2004). Empathie et expérience humaine : essai de circulation mutuelle entre science cognitive, sagesse contemplative et philosophie phénoménologique. Théologiques, 12(1-2), 39-70.

https://doi.org/10.7202/011555ar

Tous droits réservés (C) Faculté de théologie et de sciences des religions, Université de Montréal, 2005
Ce document est protégé par la loi sur le droit d'auteur. L'utilisation des services d'Érudit (y compris la reproduction) est assujettie à sa politique d'utilisation que vous pouvez consulter en ligne.

https://apropos.erudit.org/fr/usagers/politique-dutilisation/ 


\title{
Empathie et expérience humaine ${ }^{1}$
}

Essai de circulation mutuelle entre science cognitive, sagesse contemplative et philosophie phénoménologique

\author{
Evan ThOMPSON ${ }^{2}$ \\ Département de Philosophie \\ York University \\ Titulaire de la chaire de recherche du Canada \\ sur La science cognitive \\ et l'inscription corporelle de l'esprit
}

\section{Introduction}

Comment pouvons-nous comprendre la science et la religion comme surgissant de l'expérience humaine tout en la transcendant? Je tenterai de répondre à cette question à partir de mon champ d'expertise, celui du rapport entre l'expérience humaine et l'étude scientifique de l'esprit dans la science cognitive (voir Varela et al. 1991). Une des questions centrales qui m'habitent est la suivante: "Quelle forme devrait prendre une science de l'esprit humain qui aurait atteint sa maturité? " Par "science qui aurait atteint sa maturité », j'entends une science qui soit développée au point où ses chercheurs s'avèrent expérimentés et compétents vis-à-vis de leur sujet d'étude. J'estime qu'une science de l'esprit qui se prétend mature devrait inclure des «méthodes d'analyse à la première personne »

1. La version originale anglaise de ce texte paraîtra dans Proctor (2005). Nous le reproduisons avec l'aimable permission de Oxford University Press, dans une traduction de Lamphone Phonevilay, révisée par Jean Duhaime. Les citations tirées d'ouvrages en anglais ont également été traduites.

2 Je dédie cet essai à la mémoire de F.J. Varela (1946-2001), un «passeur» enthousiaste entre la science, la phénoménologie et la sagesse contemplative, dont je déplore profondément la disparition prématurée, mais qui continue d'être une source d'inspiration pour moi et pour ses collègues. 
rigoureuses pour étudier l'expérience subjective en collaborant de façon active avec les «méthodes d'analyse à la troisième personne» de la science biobehavioriste. Les «méthodes d'analyse à la première personne » sont des pratiques qui font augmenter la sensibilité d'un individu à sa propre expérience par le biais d'un entraînement systématique de l'attention et de l'autorégulation de l'émotion (voir Varela et Shear 1999; Depraz et al. 2003). Cette capacité de prêter attention à l'expérience ellemême - prêter attention non seulement à l'objet, c'est-à-dire ce qui est expérimenté par l'individu, mais également à l'acte par lequel ce dernier expérimente cet objet - semble être une habileté et un mode d'expérience exclusivement humains, qu'on ne retrouve pas chez les autres animaux. Les méthodes d'analyse de la première personne, pour cultiver cette capacité, ont été développées principalement dans les traditions contemplatives de sagesse de l'expérience humaine, en particulier dans le bouddhisme. À travers l'histoire, la religion a constitué le lieu privilégié de l'expérience contemplative et de son articulation théorique en philosophie et en psychologie. C'est pourquoi ma recherche en sciences cognitives et en philosophie de l'esprit aborde la religion non pas comme un objet d'étude scientifique (comme c'est le cas pour Boyer 2005) mais comme un espace où sont mises en œuvre des méthodes d'analyse à la première personne qui peuvent jouer un rôle actif et créatif dans la recherche scientifique elle-même (voir Lutz et Thompson 2003).

Cependant l'expérience contemplative n'est pas l'unique composante de la religion; en fait, plusieurs religions l'ignorent totalement ou ne lui font qu'une place limitée. Par ailleurs, l'expérience contemplative peut se retrouver dans des contextes non religieux importants, par exemple dans la philosophie (voir McGee 2000). Pour ces raisons, le terme "religion» ne désigne pas adéquatement cette tradition culturelle ou ce domaine de l'expérience humaine que moi-même et d'autres chercheurs désirons faire dialoguer de façon constructive avec les sciences cognitives. Les expressions «traditions de sagesse» et «expérience contemplative» seraient plus adaptées. De même, l'expression «dialogue science-religion» n'exprime pas adéquatement la nature de notre projet, puisque notre but n'est pas de nous positionner entre les affirmations de la science et celles de la religion, mais de parvenir à une meilleure compréhension de la conscience et de l'esprit humains en faisant de la psychologie contemplative une partenaire à part entière de la science de l'esprit. 
Trois champs de connaissance s'avèrent cruciaux pour cette entreprise. J'en ai déjà mentionné deux: la science cognitive et la psychologie contemplative. Le troisième est la philosophie phénoménologique dans la tradition inaugurée par Edmund Husserl. L'importance de la phénoménologie est qu'elle fournit un tiers médiateur entre la science cognitive et la psychologie contemplative, en particulier en ce qui a trait aux traditions contemplatives non occidentales tel le bouddhisme. La phénoménologie est une tradition intellectuelle qui s'enracine fortement dans la pensée scientifique occidentale, mais c'est aussi une tradition qui maintient l'importance de porter une attention rigoureuse aux phénomènes mentaux en tant qu'expériences vécues. Ainsi, au lieu du dialogue science-religion tel qu'il est habituellement présenté, la tâche dans laquelle je m'engage est d'effectuer des allers et retours entre les trois sphères que sont la science cognitive expérimentale, la phénoménologie et la psychologie contemplative. La «circulation mutuelle» est l'expression que Francisco Varela, Eleanor Rosch et moi-même avons introduite pour décrire cette approche (voir Varela et al. 1991). Selon la logique de la circulation mutuelle, chacun des domaines que sont la science cognitive, la phénoménologie et la psychologie contemplative demeure distinct et possède son propre degré d'autonomie - au plan des méthodes, des motivations et des préoccupations -, mais les trois se recoupent et partagent des zones communes. Ainsi, au lieu d'être juxtaposés, par opposition ou en tant que séparés mais égaux, ils vont et viennent l'un dans l'autre et s'enrichissent ainsi mutuellement.

Dans cet essai, j'illustre cette approche en explorant l'expérience humaine de l'empathie. J'ai choisi l'empathie parce qu'elle constitue un aspect majeur (bien que non le seul) de l'intersubjectivité de l'expérience humaine. L'intersubjectivité est importante lorsqu'il est question de discuter de la relation entre la science cognitive et l'expérience contemplative parce qu'il existe une tendance, dans ce champ, à mettre l'accent sur la conscience, comme s'il s'agissait d'un phénomène intrinsèquement " intérieur ", ou d'une "réalité intérieure ", invisible à la perception ordinaire. Je crois que cette façon de concevoir la conscience est erronée. Elle opère en utilisant les catégories chosifiées de «l'interne" et de «l'externe». Or, ces catégories sont inadéquates pour comprendre comment l'expérience humaine est constituée à la fois de notre corps vivant et de notre monde social interpersonnel. Nous voyons l'expérience de la honte dans le visage qui rougit, celle de la perplexité dans le sourcil qui se 
fronce, celle de la joie dans le visage qui sourit; nous n'appréhendons pas leur existence comme phénomènes «internes", distincts de faits «externes ". Même s'il s'avère que toutes les expériences ne peuvent être exprimées de façon corporelle, et que chacun de nous n'a un accès immédiat qu'à sa propre expérience, cela ne signifie pas que l'expérience est «intérieure" d'une façon métaphysique spéciale (et peu claire). Le fait de focaliser notre recherche sur l'empathie contribue à nous rappeler que nous avons besoin d'un meilleur cadre pour penser l'expérience humaine - que ce soit en science cognitive ou en psychologie contemplative que celui de «l'intérieur» et de "l'extérieur».

L'idée principale de la partie suivante de cet essai est que l'expérience humaine dépend de façon formative et constitutive d'un jumelage dynamique de soi et de l'autre dans l'empathie. Après avoir présenté cette idée en intégrant le point de vue de la science cognitive et celui de la phénoménologie, j'élargirai la discussion pour inclure la perspective contemplative sur la non-dualité de soi et de l'autre, telle qu'elle se présente dans le Madhyamaka, la "voie du milieu » de la tradition bouddhiste indotébétaine. Finalement, je revendrai sur l'importance de la phénoménologie contemplative pour la science cognitive.

\section{Définir l'empathie}

Il est préférable de penser d'abord l'empathie d'une façon large, pour ensuite distinguer différents types d'empathie au fur et à mesure que notre réflexion progressera. Malgré tout, même avec une définition large, il existe plusieurs façons de définir l'empathie - l'empathie comme capacité intentionnelle de base, l'empathie comme un cas unique d'acte intentionnel, et l'empathie comme processus intentionnel. (J'utilise ici le terme «intentionnel » dans son sens husserlien de «mental directedness » à l'endroit d'un objet ou d'ouverture à ce qui est autre.) Comme capacité intentionnelle, l'empathie est cette habileté de base à comprendre l'expérience d'un autre individu, une capacité qui sous-tend tous les sentiments et émotions particuliers qu'une personne peut avoir pour une autre (voir Vetlesen 1994). Faire usage de cette capacité revient à s'engager dans une relation d'empathie en tant qu'acte et processus intentionnels. Comme type particulier d'acte intentionnel, l'empathie tire son caractère intentionnel du fait qu'elle est dirigée vers l'expérience d'une autre personne (voir Stein 1964). Même si l'empathie ainsi comprise est fondée sur la 
perception sensorielle (de la présence physique de l'Autre) et peut impliquer une démarche d'inférence dans un contexte difficile ou problématique (lorsqu'on doit saisir comment une autre personne se sent à propos de quelque chose), elle ne peut être réduite à une simple addition de la perception et de l'inférence - sur le modèle des théories avançant que nous comprenons les autres en observant d'abord leur comportement corporel et ensuite en inférant ou en faisant l'hypothèse que leur comportement est causé par des expériences ou états mentaux internes similaires à ceux qui provoquent un comportement semblable chez nous. Au contraire, dans l'empathie nous expérimentons l'Autre directement comme personne, c'est-à-dire comme un être intentionnel et mental dont la gestuelle et les actions corporelles traduisent l'expérience et les états d'esprit. Finalement, la notion d'empathie, en tant que processus intentionnel, s'applique à tout processus dans lequel une perception attentive de la situation ou de l'état de l'Autre génère chez quelqu'un un état ou une situation qui s'applique davantage à l'état ou à la situation de l'Autre qu'à son propre état ou à sa propre situation antérieure (voir Preston et de Waal 2002).

Ayant formulé cette définition de l'empathie au sens large, nous pouvons maintenant identifier quelques types spécifiques d'empathie. Les psychologues utilisent le terme "empathie " pour décrire au moins trois processus différents: (1) ressentir ce qu'une autre personne est en train de ressentir; (2) savoir ce qu'une autre personne est en train de ressentir; et (3) répondre avec compassion à la douleur d'une autre personne (voir Levenson et Reuf 1992). Cependant, des phénoménologues, suite à des analyses plus détaillées au plan structurel, distinguent au moins quatre aspects principaux dans la manifestation complète de l'empathie (voir Depraz 2001):

1. Le couplage ou pairage involontaire de mon corps vivant avec ton corps vivant dans la perception et dans l'action.

2. Le mouvement ou la transposition imaginaire de ma personne à ta place.

3. L'interprétation de toi comme étant un Autre pour moi et de moi comme étant un Autre pour toi.

4. La perception éthique et morale de toi comme personne. 


\section{L'empathie comme couplage}

Le premier type d'empathie - le couplage ou pairage dynamique des corps vivants de soi et de l'autre - est de l'ordre de la perception et de l'action préréflexive (ce que Husserl [2001] appelle la "synthèse passive » de l'expérience). Cette empathie est passive dans la mesure où elle n'est pas initiée volontairement; elle sert de support aux autres types d'empathie. Le "couplage» ou le "pairage » désigne un lien associatif de soi et de l'autre sur la base de leur similarité corporelle. Cette similarité n'opère pas tant au niveau de l'apparence visuelle, qui constitue une partie de l'image corporelle comme un objet intentionnel présent à la conscience, qu'au niveau du geste, de la posture et du mouvement, c'est-à-dire au niveau du schème corporel inconscient ${ }^{3}$. Ainsi, l'empathie ne consiste pas simplement à comprendre les expériences particulières d'une autre personne (la tristesse, la joie, et ainsi de suite), mais plutôt à faire l'expérience de l'autre comme sujet d'expérience, corporel et vivant comme soi-même.

Cette conception phénoménologique de la base somatique de l'empathie peut être associée à la science cognitive si l'on retourne à la notion large de l'empathie comme processus - comme tout processus dans lequel une perception attentive de l'Autre génère chez quelqu'un un état qui s'applique davantage à l'état de l'Autre qu'à son propre état antérieur. Selon le modèle "perception-action" de l'empathie (voir Preston et de Waal 2002), lorsque nous percevons le comportement d'une autre personne, nos propres représentations motrices pour ce type de comportement sont automatiquement activées et génèrent les réponses automatiques et somatiques correspondantes (à moins qu'elles soient inhibées). Par exemple, il a été démontré que lorsqu'un individu en voit un autre exécuter des actions avec des parties différentes du corps (les actions de la bouche, de la main et du pied), les patterns neuronaux d'activation correspondent, dans le cerveau de l'observateur, à ceux qui seraient actifs s'il exécutait lui-même ces mêmes actions corporelles (voir Buccino et al. 2001).

On appelle ce type de couplage de soi et de l'autre un couplage sensori-moteur. En plus du couplage sensori-moteur, il existe un couplage affectif ou une "résonance affective» (voir de Waal 2002). Dans la

3. En ce qui concerne la distinction entre l'image corporelle et le schème corporel, voir Gallagher 1986. 
résonance affective, deux individus engagés dans une interaction directe s'affectent mutuellement au niveau leurs états émotionnels.

\section{L'empathie comme transposition imaginaire}

Le deuxième type d'empathie - l'empathie comme la transposition imaginaire de soi à la place de l'Autre - est plus actif et cognitif que le premier. Au lieu du simple pairage corporel involontaire de soi et de l'autre, des procédés de saisie de perspective cognitive sont utilisés pour s'imaginer ou se transposer mentalement à la place de l'Autre.

Des études comparatives d'éthologie cognitive sur l'empathie apportent un éclairage important sur l'empathie cognitive. La présence et l'étendue de l'empathie chez les animaux non-humains, en particulier les primates, donne lieu à de vifs débats. Selon une vision du "tout ou rien », l'empathie cognitive (le seul type d'empathie qui existe, selon cette vision) requiert la capacité cognitive d'attribuer des états mentaux à un autre individu et de comprendre le comportement de l'autre à la lumière de ceux-ci. Cette capacité, généralement appelée «lecture de la pensée ${ }^{4}$ » est considérée par certains comme nécessitant une "théorie de la pensée ", un corps de connaissances théoriques sur les états mentaux et leur rôle pour générer le comportement. Les partisans de cette façon de penser soutiennent que les chimpanzés échouent dans certains de ces tests de «lecture de la pensée » et conséquemment ne possèdent pas une théorie de la pensée, de sorte qu'ils sont incapables d'empathie cognitive. Mais, comme je l'ai indiqué ici et comme d'autres, notamment Frans de Waal, l'ont suggéré, l'empathie ne devrait pas être perçue comme étant un phénomène de tout ou rien. De Waal s'exprime ainsi à ce propos: «De nombreuses formes d'empathie se situent de manière intermédiaire entre les extrêmes que sont, en face d'un autre, soit une simple agitation et une détresse, soit une compréhension en profondeur de l'état pénible dans

4. «Mind-reading». L'expression semble pauvre pour décrire la nature fondamentale de nos habiletés cognitives intersubjectives. Cette expression suggère que nous sommes essentiellement des spectateurs vis-à-vis de l'autre, que la vie sociale humaine est basée principalement sur la capacité de "lire» les états mentaux internes comme observateur ou spectateur, sur la base d'un comportement externe (de la même manière que nous lisons la signification des mots sur la base de signes écrits). Pour une critique de cette vision, voir McGeer 2001 et Gallagher 2001. 
lequel il peut se trouver. Au premier bout du spectre, les nourrissons des rhésus deviennent irrités et recherchent le contact mutuel dès que l'un d'eux se met à crier. À l'autre bout, un chimpanzé se souvient d'une blessure qu'il a infligée et retourne voir sa victime pour l'inspecter.» (1996, 69)

Parmi les cas intermédiaires possibles, on trouve des comportements de consolation ou des comportements d'aide adaptée. Les comportements de consolation se produisent lorsqu'un agent non impliqué et moins en détresse établit un contact amical avec la victime d'une agression. Par exemple, de Waal, dans son livre Good Natured, présente la photo d'un chimpanzé juvénile en train de réconforter un adulte en détresse. Le comportement de consolation n'a été documenté abondamment que dans le cas des grands singes (on ne l'a pas observé chez les autres espèces de singes, en dépit de grands efforts pour le détecter). L'aide adaptée consiste à porter secours à un autre (qu'il s'agisse d'un membre de la même espèce ou non) avec des comportements adaptés à ses besoins particuliers (comme lorsqu'un gorille en aide un autre à descendre d'un arbre ou essaie d'aider un oiseau blessé à voler). Un tel comportement, selon de Waal, «nécessite probablement une distinction entre soi et l'autre qui permette à l'individu de dissocier la situation de l'autre de la sienne propre, tout en maintenant le lien émotionnel qui motive un tel comportement. ( (de Waal 2002). Il existe un grand nombre de cas anecdotiques d'aide adaptée chez les grands singes.

L'empathie cognitive totale, cependant, n'est atteinte que lorsqu'un individu peut mentalement adopter la perspective de l'autre en changeant de place avec lui dans son imagination. Cela pourrait se décrire phénoménologiquement ainsi ${ }^{5}$ : je suis ici et j'imagine que je vais là, à la place où tu es présentement. Inversement, tu es ici (ce là où j’imagine être) et tu imagines que tu vas là, à la place où je suis (mon ici). À travers ce mouvement imaginaire et cette transposition spatiale, nous sommes capables d'échanger nos perspectives mentales, nos pensées et émotions. Il n'est pas évident que les grands singes possèdent ce type de capacité mentale; la question est débattue (voir Gallup 1998; Povinelli 1998).

Chez les enfants humains, la capacité à se transposer mentalement à la place de l'autre semble être liée à l'émergence, vers l'âge de neuf à

5. Cette description s'inspire de Depraz 2001, 173. 
douze mois, d'un ensemble complet d'habiletés cognitives communément appelées «attention conjointe» (voir Tomasello 1999, 62-63). La notion d' "attention conjointe" fait référence à la structure triadique d'un enfant, d'un adulte, et d'un objet ou événement sur lequel porte leur attention $^{6}$; elle inclut des activités qui consistent à suivre le regard (regarder exactement au même endroit que les adultes), à interagir avec un même objet ou dans un même événement, à utiliser les adultes comme points de référence sociale et à apprendre par imitation (agir avec les objets comme le font les adultes). Au cours de la même période environ, le nourrisson commence également à pointer des choses du doigt et à les tendre à quelqu'un pour les lui montrer; ces gestes servent à attirer activement et intentionnellement l'attention des adultes. Michael Tomasello soutient que les «nourrissons commencent à entrer dans des interactions d'attention conjointe lorsqu'ils commencent à comprendre que les autres sont des agents intentionnels comme soi » (Tomasello 1999, 68). Il suggère que cette étape marquante du développement cognitif du nourrisson se produit grâce à une «simulation» en vertu de laquelle le nourrisson combine sa compréhension primaire des autres en tant que "comme moi» (ce qui est le processus fondamental de l'empathie comprise en termes phénoménologiques) et la nouvelle compréhension qu'il a de luimême en tant qu'être capable d'agir intentionnel; il s'en sert comme une base à partir de laquelle il juge analogiquement et catégoriquement que les autres sont aussi des agents intentionnels «comme moi ».

\section{L'empathie comme compréhension de Toi comme un Autre pour Moi, et de Moi comme un Autre pour Toi}

Le troisième type d'empathie n'implique pas seulement le fait de m'imaginer à ta place, mais de te comprendre comme un Autre qui en retour me voit comme étant un Autre pour toi. En d'autres mots, la transposition imaginaire, dans ce type d'empathie, implique la possibilité de me voir à partir de ta perspective, c'est-à-dire de la façon que tu m'expérimentes empathiquement. L'empathie devient ainsi réitérée, de telle sorte que j'imagine empathiquement ton expérience empathique de moi, et que

6. N.D.L.R. Voir l'article de Sonia Mansour-Robaey et Philippe Robaey dans ce numéro. 
tu imagines empathiquement mon expérience empathique de toi. Nous nous parlons également de nos expériences, et ainsi la communication et l'interprétation linguistiques participent à cet échange et le structurent. Le résultat est que chacun de nous développe un point de vue intersubjectif qui transcende nos perspectives singulières de première personne.

Nous pouvons encore une fois faire appel à la psychologie développementale pour avoir un aperçu de la genèse de ce troisième type d'empathie et du rôle qu'il joue dans la constitution d'une perspective intersubjective. Permettez-moi de citer un passage du livre de Tomasello, The Cultural Origins of Human Cognition, qui décrit avec lucidité cette genèse chez le nourrisson:

Lorsque le nourrisson de neuf à douze mois commence à suivre et à diriger l'attention des autres vers des objets externes, il arrive parfois que l'autre personne dont l'attention est dirigée par le nourrisson fixe ce dernier. Le nourrisson dirige alors l'attention de cette personne envers lui-même d'une manière qui n'était pas possible antérieurement, à savoir, avant la révolution socio-cognitive qui a lieu vers neuf mois. À partir de ce moment-là, les interactions face-à-face que le nourrisson a avec les autres — même si elles sont apparemment en continuité avec les interactions face-à-face de la petite enfance - sont radicalement transformées. Il sait alors qu'il est en interaction avec un agent intentionnel qui le perçoit et a des intentions à son endroit. Tant que le nourrisson ne comprenait pas que les autres perçoivent et ont des intentions envers le monde extérieur, il n'était pas question de se demander comment ils me percevaient et avaient des intentions envers moi. Mais une fois que le nourrisson a compris cela, il peut gérer la relation intentionnelle de l'adulte vis-à-vis du monde, s'incluant luimême [...]. Grâce à un processus similaire, le nourrisson de cet âge devient également capable de gérer les attitudes émotionnelles des adultes envers lui - une manière de donner une connotation sociale à l'attitude des autres envers soi. Cette nouvelle compréhension de la manière dont les autres se sentent par rapport à moi rend possible le développement de la gêne, de la conscience de soi et d'un sens de l'estime de soi [...]. On en veut pour preuve le fait que, quelques mois après la révolution socio-cognitive, à son premier anniversaire, le nourrisson commence à montrer les premiers signes de gêne et de timidité devant les autres personnes et les miroirs... (Tomasello 1999, 89-90)

Comme l'explique ensuite Tomasello, une fois que le nourrisson comprend les autres individus comme étant des êtres intentionnels et se comprend lui-même comme étant un participant parmi d'autres dans une 
interaction sociale, de toutes nouvelles dimensions cognitives surgissent. L'enfant devient capable de participer à des scènes d'attention conjointes - des interactions sociales dans lesquelles l'enfant et l'adulte prêtent attention conjointement à un troisième objet, de même qu'à leur attention respective envers ce troisième objet, pour une période de temps prolongée, et dans lesquelles l'enfant peut conceptualiser son propre rôle avec la même perspective "externe" que l'autre personne. Les scènes d'attention conjointes, en retour, fournissent le cadre pour l'acquisition du langage et d'autres sortes de conventions de communication (voir Tomasello 1999, chap. 4).

Même si Tomasello n'utilise pas le terme «empathie» dans ce contexte, l'acte cognitif qu'il décrit comme la capacité de se conceptualiser soi-même à partir de la perspective d'une autre personne correspond à ce que les phénoménologues appellent "l'empathie réitérée ». Dans l'empathie réitérée, je me vois moi-même à partir de la perspective d'un autre et, par conséquent, je m'appréhende moi-même comme un individu dans un monde intersubjectif.

L'étude de Tomasello à propos de l'élaboration d'une telle perspective intersubjective chez l'enfant souligne la progression développementale qui va de la compréhension de l'autre comme être animé chez le nouveauné, à une compréhension de l'autre comme agent intentionnel avec un comportement d'attention et d'orientation vers un but chez le nourrisson, pour parvenir, chez l'enfant de quatre ans, à la compréhension de l'autre comme agent mental avec des pensées et des croyances (qui n'ont pas besoin d'être extériorisées dans le comportement et qui peuvent ne pas correspondre à la réalité).

Les phénoménologues, sans négliger les aspects intentionnels et mentaux du soi, attirent l'attention sur l'ambiguïté du corps vivant dans l'empathie réitérée. Le corps vivant est ce qui est le plus intimement moi ou mien, mais c'est aussi un objet de l'Autre. Parce qu'il est si intimement $m o i$, mon corps ne peut pas se tenir devant moi comme un objet, de la même façon que les autres choses. Peu importe comment je me tourne, mon corps est toujours $i c i$, au point zéro de mon espace égocentrique, jamais là. C'est à travers le fait d'appréhender empathiquement la perception que l'Autre a de moi-même que je suis capable d'appréhender mon propre corps vécu comme un objet appartement à un monde intersubjectif. De cette façon, mon sentiment d'avoir une identité propre dans le monde, même au niveau primaire de la corporéité, est inséparable 
d'une reconnaissance par un autre, et de la capacité d'appréhender empathiquement cette reconnaissance.

\section{L'empathie comme la perception éthique et morale de toi comme personne}

Le quatrième type d'empathie est la reconnaissance de l'Autre comme personne qui mérite de l'attention et du respect. L'empathie dans ce sens ne doit pas être identifiée avec un sentiment particulier de souci pour l'autre, comme la sympathie, l'amour, ou la compassion, mais plutôt comme la capacité sous-jacente d'avoir de tels sentiments de préoccupation envers l'autre (voir Vetlesen 1994).

Ce type d'empathie peut aussi être présenté à partir d'une perspective développementale. Comme nous l'avons vu, il y a une progression qui va de la compréhension des autres comme agents intentionnels chez le nourrisson (avec de l'attention, des stratégies comportementales et des buts) à une compréhension des autres comme agents mentaux (avec des croyances, des désirs et des plans) chez le jeune enfant. Selon Piaget et Tomasello, la compréhension morale commence à apparaître environ en même temps que l'enfant en vient à comprendre les autres comme étant des agents mentaux. Cela ne provient pas des règles que les adultes imposent au comportement de l'enfant, mais du fait d'éprouver de l'empathie envers les autres personnes comme agents mentaux et d'être capable de voir et de sentir les choses de leur point de vue (voir Tomasello 1999, 179-181).

Dans la philosophie morale occidentale, il existe une longue tradition, remontant à Emmanuel Kant, qui privilégie la raison à l'émotion. Agir selon la raison est considéré comme ayant une plus grande valeur morale que d'agir sur la base de l'émotion ou du sentiment. Pourtant, comme l'observe Frans de Waal, faisant écho à David Hume et à Adam Smith: "L'aide envers ceux qui sont dans le besoin ne serait jamais intériorisée en tant que devoir sans le sentiment qui incite les gens à s'intéresser aux autres. Les sentiments moraux viennent en premier, les principes moraux en second.» $(1996,87)$

L'empathie est la capacité cognitive et émotionnelle de base soustendant toutes les émotions et tous les sentiments moraux qu'une personne peut éprouver à l'égard d'une autre. Ce qui est en question ici n'est pas que l'empathie épuise l'expérience morale, ce qui n'est évidemment 
pas le cas, mais plutôt que l'empathie fournit la source de ce genre d'expérience et son point d'entrée. Sans l'empathie, le souci et le respect pour les autres dans le sens moral - comme fins en soi - seraient impossibles. Comme Mark Johnson le soutient:

L'impératif kantien de toujours considérer les autres (et soi-même) comme étant des fins en soi n'a aucune signification pratique si l'on ne s'imagine pas à la place de l'autre. Contrairement aux prétentions explicites de Kant, nous ne pouvons pas savoir ce que signifie traiter quelqu'un comme fin en soi de façon concrète à moins de pouvoir imaginer son expérience, ses sentiments, ses buts et espoirs. Nous ne pouvons pas savoir ce que le respect envers autrui exige de nous sans participer imaginairement à leur expérience du monde. (1993, 200)

Les quatre aspects ou types d'empathie que j'ai présentés ne sont pas indépendants, mais apparaissent ensemble dans l'expérience du face-àface intersubjectif. Ils s'entremêlent à travers le corps vivant et à travers le langage. Tu t'imagines toi-même à ma place sur la base de la similarité expressive et du couplage spontané de nos corps vivants. Ton expérience contribue à la constitution de moi pour moi-même, car je m'expérimente moi-même comme un être intersubjectif en imaginant de façon emphatique ton expérience empathique de moi. Inversement, je m'imagine moimême à ta place, et mon expérience contribue à la constitution de toi pour toi-même. En communiquant par le langage et les gestes, nous nous interprétons et nous comprenons l'un l'autre dialogiquement. Cette dynamique dialogique n'est pas une combinaison linéaire ou additive de deux esprits préexistant liées à leur crâne. Elle émerge du couplage non linéaire de soi-même et de l'autre et le façonne, dans la perception et l'action, l'émotion et l'imagination, le geste et la parole. C'est cette image que j'avais en tête plus tôt lorsque j'ai dit que l'expérience humaine dépend du couplage dynamique de soi et de l'autre dans l'empathie.

\section{La non-dualité de Soi et de l'Autre ${ }^{7}$}

Pour évaluer la profondeur expérientielle et les possibilités développementales de l'empathie, nous devons l'aborder du point de vue de la psychologie contemplative. La psychologie contemplative bouddhiste est

7. N.D.L.R. Voir l'article de Victor Sōgen Hori dans ce numéro. 
particulièrement significative pour cette étude en raison de la manière dont elle combine les pratiques contemplatives de l'empathie, à la première personne, avec une vision philosophique de la non-dualité de soi et de l'autre.

Pour le besoin de la cause, je prends comme point de référence le texte classique La voie du Bodhisattva (Bodhisattvacharyavatara) du philosophe indien du viII ${ }^{\mathrm{e}}$ siècle Shantideva (1997). Selon le système philosophique bouddhiste (l'école de Prasangika Madhyamaka ou de "La voie du milieu»), Shantideva affirme que «le soi » et «l'autre » n'existent pas de façon indépendante et n'ont pas d'identité intrinsèque, mais existent seulement sur la base d'une imputation conceptuelle ou mentale. Un célèbre commentaire tibétain explique:

Même s'ils n'ont aucune raison ultime d'agir ainsi, tous les êtres pensent en termes de «je» et de «mien». À cause de cela, ils conçoivent «l'autre», qu'ils pensent comme quelque chose d'étranger; cela n'a pas de fondement non plus. Outre le fait d'être de simples imputations mentales, le «je» et «l'autre» sont totalement irréels. Ils relèvent tous les deux de l'illusion. De plus, lorsqu'on réalise la non-existence du «je », la notion de «l'autre» disparaît également, et ce, pour la simple raison que ces deux termes ne sont posés qu'en relation l'un à l'autre. Tout comme il est impossible de couper le ciel en deux avec un couteau, de la même manière, lorsqu'on réalise la dimension quasi spatiale de cette absence de contours du soi, il n'est plus possible de faire une séparation entre le «je » et «l'autre »; il en surgit l'attitude de désirer protéger les autres comme soi-même, et de protéger tout ce qui leur appartient avec le même soin qu'on mettrait à s'occuper de ses propres biens. Comme il est dit, "quiconque met de côté la vision ordinaire et triviale du "soi" découvrira la signification profonde d'un "soi totalisant" ». (Shantideva 1997, 180-181)

En affirmant que le soi et l'autre n'ont d'existence qu'en tant qu'imputations mentales, il ne s'agit pas d'être nihiliste. Les philosophes du Madhyamaka adoptent une voie moyenne entre le nihilisme et l'absolutisme, et conséquemment distinguent entre deux genres de vérité - la vérité conventionnelle et la vérité ultime. Selon la vérité conventionnelle, des individus comme toi et moi existons, ce qui écarte le nihilisme. Selon la vérité ultime, d'autre part, il n'y a pas d'«ego» ou de "je» qui existe et qui soit identifiable intrinsèquement (et, par conséquent, il n'y a pas non plus d' "autre» ou d' "alter ego » qui existe et qui soit identifiable intrinsèquement), ce qui écarte l'absolutisme. La voie du milieu est la 
vérité ultime qui attribue au «soi» une origine dépendante: il est le produit de causes et de conditions préalables, de processus mentaux et physiques en transformation constante, et d'imputations conceptuelles du «je» et de «l'autre» sur la base de ces processus mentaux et physiques. Malgré tout, en tant qu'êtres non illuminés, nous croyons par erreur, à un niveau émotionnel profond, qu'il existe un vrai «je» ou «ego » à l'intérieur de notre esprit et de notre corps, et, par conséquent, notre expérience de nous-mêmes et des autres est profondément égocentrique. Selon le Madhyamaka, et en fait selon toutes les écoles bouddhistes, c'est cet attachement égocentrique à un soi mentalement imputé qui est la vraie source de toute souffrance. L'illumination consiste, dit-on, en l'éradication de cet égocentrisme à sa source même, de telle sorte que l'expérience de l'individu ne soit plus gouvernée par cet attachement à soi-même.

Il y a évidemment des différences significatives entre cette vision philosophique et la phénoménologie. Ce qui me préoccupe ici, cependant, ce ne sont pas ces différences importantes et intéressantes, mais plutôt le rôle similaire que l'imagination empathique active joue dans les deux traditions pour décentrer l'ego et ainsi ouvrir l'expérience humaine à une intersubjectivité originaire avant les imputations mentales réifiées du «soi » et de «l'autre».

Dans le huitième chapitre de son texte, Shantideva présente deux méditations, la méditation sur l'égalité du soi et de l'autre, et la méditation sur l'échange du soi et de l'autre. Dans la première méditation sur l'égalité du soi et de l'autre, la personne commence par la conviction égocentrique que "ceci est mon soi", puis réalise, en réfléchissant de façon critique, que «mon soi » est tout simplement un nom attribué à une collection d'éléments physiques et mentaux. La personne impose mentalement les qualifications intrinsèques d'un «je» et d'un «autre» aux phénomènes, mais le «je » et l' "autre» sont simplement des désignations relatives imputées à des éléments en qui ce «je » et cet "autre» n'existent pas de façon inhérente. Chaque «je» est un «autre», et chaque "autre» est un «je». Tous les êtres sont exactement dans la même situation qui leur fait imputer les qualificatifs de "mien» et d' "autre", et tous sont aux prises avec la même préoccupation d'être heureux et de ne pas souffrir. Ayant pris conscience de l'égalité du soi et de l'autre, la personne en arrive à visualiser les souffrances des autres êtres comme étant les siennes. Le commentaire tibétain cité plus haut formule cela ainsi: 
Selon ces enseignements, en qualifiant de «je» la totalité des êtres souffrants, et en s'habituant à penser constamment qu'ils sont "moi-même ", le «je» se sensibilise de plus en plus à ces êtres et en vient à se soucier autant d'eux que de soi-même [...]. Par rapport à la souffrance comme telle, la distinction entre la souffrance des autres et ma souffrance est insignifiante. Il s'ensuit que, même si la douleur d'autrui ne m'affecte pas directement, malgré tout, si j'identifie cet autre comme «je» ou «mien», la souffrance de cet autre me devient également insupportable. (Shantideva 1997, 182)

Faire l'exercice de cette première méditation sur l'égalité entre le soi et l'autre est un prérequis essentiel à la deuxième méditation sur l'échange de soi et de l'autre. Dans cette seconde méditation, à travers l'imagination empathique et sympathique, la personne se visualise dans la position des autres et s'imagine comment elle apparaît à leur regard. Cette méditation exploite explicitement des émotions négatives spécifiques, ou des "facteurs mentaux» malsains, comme on les appelle dans le bouddhisme ${ }^{8}$. Ces émotions sont la fierté, la rivalité compétitive et la jalousie. Une personne ressent de la fierté devant une personne inférieure, de la rivalité compétitive devant quelqu'un qui lui est égal, et de la jalousie devant un supérieur. Comme antidote à ces émotions, la personne se regarde elle-même avec les yeux de quelqu'un d'inférieur, d'égal ou de supérieur, et génère l'émotion correspondante envers elle-même, de telle sorte qu'elle comprend ce que cela signifie d'être l'autre partenaire de la relation. Par exemple, l'expérimentation empathique de l'envie d'un inférieur envers soi-même et la souffrance qu'elle implique est l'antidote à la fierté. En même temps, la personne assume les souffrances de ces autres comme étant les siennes propres (ce à quoi l'aura préparé la méditation sur l'égalité entre le soi et l'autre).

La méditation sur l'échange entre le soi et l'autre est ainsi une forme contemplative disciplinée d'empathie réitérée. Par «disciplinée» je ne veux pas seulement dire que la méditation est un exercice de visualisation par étapes. Elle est disciplinée également parce qu'elle nécessite pour sa réalisation - tout comme la première méditation sur l'égalité entre le soi et l'autre - les pratiques contemplatives bouddhistes fondamentales de

8. Pour une discussion des rapports entre le concept occidental d' "émotion" et le concept bouddhiste de «facteurs mentaux», voir Dreyfus 2002. 
la stabilité attentionnelle (shamatha) et "la conscience pénétrante» (vipashyana). Pour réaliser la visualisation, la personne a besoin de garder son esprit concentré de façon soutenue sur l'image de l'autre comme «je» et sur l'image de soi-même telle que cet «alter ego" la perçoit; il lui faut également une conscience pénétrante de la myriade de phénomènes mentaux et physiques qui surgissent à chaque instant dans le champ de l'expérience intersubjective.

Du point de vue de la science cognitive, la méditation sur l'égalité entre le soi et l'autre de même que celle sur l'échange entre le soi et l'autre sont remarquables par la manière disciplinée dont elles combinent les méthodes à la première personne concernant la stabilité attentionnelle, la visualisation et l'imagerie mentale, avec la modulation cognitive de l'émotion'. Du point de vue phénoménologique, elles sont remarquables par la manière disciplinée dont elles utilisent la technique phénoménologique clé de la "variation imaginative » qui consiste à varier librement les phénomènes dans l'imagination afin de discerner leurs formes invariantes.

La philosophie Madbyamaka sous-jacente à ces méditations se prête également à une comparaison avec l'analyse phénoménologique de l'intersubjectivité en termes d' "ipséité » et d' "altérité », ou de perception du «je» et de "l'autre» (voir Zahavi 1999; 2001). Ce niveau est plus profond que l'analyse en termes d'empathie, et il démantèle radicalement la perspective égocentrique d'une manière semblable à celle du Madhyamaka.

Selon la phénoménologie, l'altérité ou perception de l'autre appartient à la structure de l'expérience avant même qu'une rencontre empathique ait lieu. L'empathie fait réaliser l'altérité en étant une expérience de décentrement ou de déplacement de soi. Dans l'empathie, je m'imagine moi-même comme autre — et, dans l'empathie réitérée, je deviens autre à moi-même en me regardant moi-même à travers les yeux

9. Il vaut la peine de préciser que le contrôle de l'attention et de la cognition, l'imagerie mentale et l'émotion étaient les trois champs d'investigation retenus pour le colloque tenu au Massachusetts Institute of Technology (Kresge Auditorium), les 13-14 septembre 2003, sous le thème «Investigating the Mind: Exchanges between Buddhism and the Biobehavioral Sciences on How the Mind Works ", auquel participaient sa Sainteté le Dalaï-Lama et un groupe de scientifiques cognitifs et de spécialistes bouddhistes. Voir $<\mathrm{http}$ ://www.investigatingthemind.org $>$. 
d'un autre. La même dynamique de substitution de soi à l'autre se manifeste par la médiation de l'expérience. Elle se produit dans l'expérience corporelle lorsqu'une main en touche une autre, et que les deux alternent et s'entremêlent dans leurs rôles de sentir et d'être senties. La substitution de soi à l'autre se produit lorsque je me rappelle mon moi passé, lorsque je réfléchis sur les expériences qui viennent de m'arriver, et lorsque je m'imagine moi-même. Ces expériences de déplacement de soi indiquent que le «je» et «l'autre» ne sont pas simplement corrélatifs et interchangeables, comme les perspectives spatiales d' «ici» et de «là », mais que la perception de soi est déjà constituée intérieurement par la perception de l'autre. L'expérience est intrinsèquement intersubjective dans le sens que l'altérité et l'ouverture à l'Autre sont des caractéristiques a priori de la structure formelle de l'expérience. Par conséquent, la présomption clé de l'égocentrisme - que la subjectivité peut s'affirmer comme ego et ainsi exclure l'Autre - vole en éclat ${ }^{10}$.

10. La résonance entre la non dualité du soi et de l'autre selon le Madhyamaka, d'une part, et l'interaction entre l'ipseité et l'altérité selon la phénoménologie husserlienne, d'autre part, mériteraient d'être explorées plus en détails qu'il n'est possible de le faire ici. Permettez-moi de faire cependant une observation dans la perspective de discussions futures. Même s'il existe un parallèle fascinant entre ces deux traditions en ce qui concerne la dépendance réciproque du «soi » et de l' "autre », elles semblent toutefois diverger dans leur façon d'envisager le "je» ou l'ego. Alors que le Madhyamaka affirme que le soi est une imputation mentale à l'endroit de phénomènes physiques et mentaux transitoires, Husserl affirme quant à lui qu'il existe un « ego pur» qu'il conçoit comme étant un pôle identitaire qui transcende tout acte d'attention particulier et qui est partagé par toutes les expériences qui appartiennent au même niveau de conscience. Je fais remarquer que même si l'ego pur husserlien équivaut somme toute au genre de notion du soi que rejette la philosophie du Madhyamaka, il ne devrait pas être considéré comme étant une version non critique ou précritique de cette notion, car Husserl a introduit l'ego pur précisément en relation avec cette structure du soi et de l'autre constitutive de la subjectivité. Comme l'écrit D. Zahavi: "La subjectivité acquiert une conscience explicite de son “je” seulement dans le rapport entre son soi et l'autre. [...] La notion husserlienne d'ego pur ne peut pas être considérée uniquement comme une manifestation et une confirmation de son adhésion à une métaphysique de la présence, puisque Husserl a introduit l'ego pur au moment où il a commencé à prendre au sérieux les actes intentionnels caractérisés par la division du soi, l'absence à soi et l'aliénation du soi.» $(1999,150)$ Il se peut que cet aspect de la phénoménologie de Husserl s'apparente à l'Advaita Vedanta plus qu'au Madhyamaka. Sur ce rapprochement, voir Gupta 1998. 
Nous avons maintenant vu comment la phénoménologie aussi bien que la psychologie contemplative transcendent l'expérience égocentrique en révélant une intersubjectivité originaire avant les conceptions réifiées de soi et de l'autre. Dans la phénoménologie de Husserl, cette transcendance de l'égocentrisme reste principalement dans la sphère théorique et cognitive, mais d'autres phénoménologues, comme Max Scheler et Emmanuel Levinas, l'appréhendent plutôt dans la sphère affective et éthique $^{11}$. La psychologie contemplative bouddhiste apporte une contribution majeure à la question en montrant comment le théorique, le cognitif, l'affectif et l'éthique peuvent être articulés ensemble grâce au recours discipliné à des méthodes à la première personne.

\section{La science cognitive contemplative et le dialogue entre science et religion}

Rappelons notre question de départ: "Comment pouvons-nous comprendre la science et la religion comme surgissant de l'expérience humaine tout en la transcendant?» Pour conclure cet essai, j'aimerais aborder cette question à la lumière de l'importance qu'a, pour le renouvellement d'une science de l'esprit, le recours aux méthodes à la première personne et à l'expérience contemplative.

La notion de transcendance est fondamentale ici. Les phénoménologues comprennent la transcendance comme une structure dynamique de l'expérience - l'expérience vise un au-delà d'elle-même et est toujours déjà ouverte à une altérité. Les phénoménologues insistent aussi sur le fait que la science est elle-même une forme d'expérience humaine. L'expérience scientifique vise clairement à transcender l'expérience ordinaire, dans le sens d'expérience préscientifique. Les modes phénoménologique et contemplatif d'investigation de l'esprit poursuivent des objectifs similaires de transcendance: les deux visent à transcender une expérience non réfléchie ou inconsciente. Cela dit, comment ce mouvement de transcendance doit-il être compris?

Pour aborder la question, permettez-moi de simplifier et d'idéaliser la pratique scientifique sous la forme de la «stratégie $\mathrm{ABC}$ » suivante,

11. Pour une étude importante sur la relation entre E. Levinas et le Prasangika Madhyamaka, voir Pitkin 2001. 
dans laquelle le but est d'aller de A à C en passant par B (voir Marbach 1998):

$\mathrm{Du}$ :

A. Niveau de cognition ordinaire (préscientifique) des phénomènes réels à l'étude,

Via :

B. La cognition, basée sur l'imagination, des phénomènes comme "possibilités pures» sujettes à des lois invariables,

$\mathrm{Au}$ :

C. Niveau de la cognition scientifique des phénomènes réels, grâce à l'application des perspectives découvertes au niveau $\mathrm{B}$.

L'exemple classique est celui de Galilée: en inaugurant le déplacement de la physique aristotélicienne vers la physique moderne, il a utilisé l'outil mathématique pour élaborer une théorie (niveau C) sur la chute des corps (niveau A) en la considérant (niveau B) comme une des nombreuses possibilités des phénomènes régis par des lois.

Supposons que nous appliquons ce modèle à la science cognitive et à sa tentative de comprendre l'expérience humaine consciente. La stratégie courante en science cognitive a été de tenter de passer d'une cognition ordinaire (préscientifique) de l'expérience consciente à une cognition scientifique en se basant principalement (niveau B) sur l'observation à la troisième personne et l'utilisation de modèles fonctionnels. En d'autres mots, il n'y a pas eu d'effort soutenu, au niveau B, pour chercher les structures invariantes de l'expérience elle-même, telle qu'elle est vécue à la première personne. Un tel effort nécessiterait le recours à des méthodes contrôlées d'analyse à la première personne. La force de cette analogie est de suggérer que la science cognitive a besoin d'incorporer dans sa recherche des méthodes d'analyse à la première personne (voir Lutz et Thompson 2003).

Les méthodes d'analyse à la première personne visent à transcender l'expérience ordinaire, non pas en la laissant derrière, mais en cultivant une forme d'éveil plus élevée ou plus intense. Voici les caractéristiques génériques de base des méthodes d'analyse à la première personne, qu'on retrouve en phénoménologie et dans la tradition contemplative de la méditation d'éveil de la conscience (shamatha-vipashyana — voir Depraz et al. 1999; Depraz et al. 2003): 
1. Suspension. On suspend les idées, croyances et préjugés préconçus sur l'expérience. On induit une attitude de «suspension» en regard de ces derniers.

2. Réorientation. On oriente l'attention non seulement vers le contenu de l'expérience (le «quoi»), mais vers le processus de l'expérience lui-même et sur son caractère de phénomène vécu un instant à la fois (le «comment»).

3. Intimité. Grâce à la suspension et à la réorientation, on accroît l'intimité ou la familiarité avec l'expérience (on utilise aussi pour ce faire des techniques additionnelles comme la variation imaginative).

4. Entraînement. Le savoir-faire et la dextérité dans le domaine s'acquièrent par un entraînement à long terme.

Les pratiques ayant ces caractéristiques sont importantes pour la science cognitive pour plusieurs raisons. Premièrement, elles aident les sujets à accéder à des aspects de leur expérience qui demeureraient autrement inaperçus, tels que l'état affectif passager ou la qualité de l'attention. Deuxièmement, les comptes rendus tout en nuances qui sont produits de cette façon par les sujets peuvent aider les expérimentateurs externes à comprendre des processus physiologiques qui, autrement, demeureraient opaques, comme la variabilité dans de la dynamique cérébrale observée lors d'expériences d'imagerie mentale (voir Lutz et Thompson 2003). Par exemple, les méthodes d'analyse à la première personne ont été utilisées pour révéler des différences phénoménologiques importantes dans la qualité subjective de l'attention pendant la perception visuelle, et ces différences ont été mises en corrélation avec des fréquences et des patterns de synchronie de phase distincts dans la dynamique de l'activité à grande échelle du cerveau, mesurée en millisecondes (voir Lutz et al. 2002). Finalement, les individus qui peuvent générer des états mentaux spécifiques et rendre compte de ces états mentaux avec un haut degré de précision phénoménologique, tels que les contemplatifs, ouvrent une voie à l'étude de l'efficacité causale des processus mentaux, considérés neurodynamiquement comme des processus globaux ou à grande échelle susceptibles de modifier les événements neuraux et somatiques locaux ${ }^{12}$.

12. Sur cette conception des états mentaux comme états neurodynamiques globaux efficaces causalement, voir Thompson et Varela 2001. 
La science cognitive commence à peine à s'ouvrir aux méthodes d'analyse à la première personne; il est donc trop tôt pour envisager tout ce qui pourrait être accompli grâce à la circulation mutuelle entre la science cognitive, la phénoménologie et la psychologie contemplative. Jusqu'à maintenant, la science cognitive n'a exploré qu'un petit segment de l'esprit humain - celui qui est accessible à des sujets qui sont naïfs au plan phénoménologique, tout comme les scientifiques du domaine cognitif auxquels il font leurs comptes rendus. La rencontre entre la phénoménologie, la psychologie contemplative et la science cognitive permet d'entrevoir une nouvelle possibilité, celle d'individus dotés d'une grande compétence en phénoménologie rendant compte de leurs expériences à des scientifiques cognitifs ayant des connaissances phénoménologiques adéquates. La perspective d'une telle collaboration et illumination mutuelle de la science cognitive, de la phénoménologie et de la psychologie contemplative renvoie à une autre sorte de transcendance, autant pour la science que pour la religion. Pour la science cognitive, cela signifie qu'on transcende le rejet positiviste de l'expérience, tandis que pour la religion, cela signifie qu'on transcendance le dogme et la croyance préscientifique. Dans les deux cas, la clé d'une telle transcendance est de faire de la psychologie contemplative et de la phénoménologie des partenaires à part entière dans la science de l'esprit.

\section{Conclusion}

Pour conclure, permettez-moi de dégager quelques-unes des implications de cette conception de la science de l'esprit pour le dialogue plus large entre la science et la religion. Comme je l'ai mentionné au début de cet essai, la circulation mutuelle entre la science cognitive et les traditions de contemplation ou de sagesse ne cadre pas facilement avec les modèles habituels de dialogue entre la science et la religion. Nous pouvons nous en rendre compte en précisant la distinction entre la perspective de circulation mutuelle et les principales autres positions adoptées dans le dialogue entre science et religion, en particulier lorsque ce dialogue porte sur la nature de l'esprit humain.

Premièrement, explorer la circulation mutuelle entre la science de l'esprit et l'expérience contemplative se distingue d'une approche qui voit la science et la religion comme étant des «magistères qui ne se chevauchent pas» (voir Gould 1999). Cette stratégie, qui isole la science et la 
religion comme deux entités indépendantes d'égale valeur, est très problématique. Elle établit une division entre la science et la religion sur la base d'un dualisme sujet-objet: la science aborde le monde empirique conçu comme le domaine de l'objectivité, alors que la religion aborde le domaine subjectif des intentions, du sens et de la valeur humaine. Comme j'ai essayé de l'illustrer dans cet essai, toutefois, ce dualisme sujet-objet ne tient pas devant l'intersubjectivité de l'expérience humaine. L'expérience intersubjective est le terrain commun de la science et de la religion, et sa compréhension s'appauvrit lorsqu'on la fracture le long d'une ligne de démarcation entre le sujet et l'objet (ou entre le fait et la valeur — voir Wallace 2005).

Deuxièmement, l'approche de circulation mutuelle se distingue d'une approche qui cherche les corrélats physiologiques des expériences religieuses (voir Newberg et al. 2001). La différence clé est que les contemplatifs ne sont pas de simples sujets expérimentaux, mais des collaborateurs et des partenaires scientifiques (voir Lutz et Thompson 2003). L'approche de la circulation mutuelle nous permet donc d'envisager que de futurs scientifiques cognitifs soient formés à la phénoménologie contemplative ainsi qu'aux techniques d'imagerie mentale et de modélisation mathématique, et que de futurs praticiens de la contemplation possèdent des connaissances suffisantes en neurosciences et en psychologie expérimentale. La science et la sagesse contemplative pourraient ainsi se délimiter et s'enrichir mutuellement. C'était précisément cette perspective que William James avait envisagée il y a plus d'un siècle, dans ses écrits sur la psychologie scientifique et l'expérience religieuse (voir Taylor 1996).

Troisièmement, l'approche de circulation mutuelle se distingue d'une vision selon laquelle la religion peut être complètement expliquée et appréhendée par la psychologie évolutive. Les travaux de Pascal Boyer $(2001$; 2005) reflètent bien cette vision; on s'y reportera pour voir en quoi son projet est différent de celui que je propose ici.

Contrairement à ceux qui voient science et religion comme des magistères qui ne se chevauchent pas, je crois qu'il est éclairant d'étudier la religion, comme le fait Boyer, à partir des perspectives de la science cognitive et de la théorie évolutive. Boyer propose de fort intéressantes analyses qui associent les concepts religieux à la compréhension intuitive que nous avons des agents, des relations sociales et des malheurs. Toutefois, en focalisant sur les structures de croyances de la religion populaire, Boyer néglige un aspect important de la religion, à savoir le fait que 
la religion (ou du moins certaines traditions religieuses) soit le principal dépôt culturel de l'expérience contemplative et des pratiques d'analyse à la première personne concernant l'expérience humaine. Boyer considère «les notions et normes religieuses » ou «les concepts religieux " comme des objets scientifiques, comme quelque chose qui a une existence autonome dans le monde; son projet est de les scruter et de les expliquer en utilisant les méthodes d'analyse à la troisième personne des sciences cognitives fonctionnalistes et évolutionnistes. En ce qui me concerne, j'envisage aussi bien le rôle que l'expérience contemplative peut jouer dans une science de l'esprit enrichie par la phénoménologie - une science de l'esprit qui inclut des modes d'investigation phénoménologiques à la première et à la deuxième personnes, en plus des modes biobehavioristes d'analyse à la troisième personne - que le rôle qu'une telle science de l'esprit renouvelée peut jouer pour faciliter la pratique de formes de l'expérience contemplative (ou plus largement de la «spiritualité») adaptées à une culture scientifique pluraliste et non-sectaire.

Il est intéressant de considérer comment l'approche de Boyer vis-àvis de la religion pourrait également s'appliquer à la science. Il en résulterait une anthropologie des structures de croyances scientifiques populaires. On pourrait demander à des gens ce qu'ils croient concernant les «gènes ", les "trous noirs ", les «réseaux neuronaux» et ainsi de suite, et ensuite étudier les liens entre ces concepts et d'autres concepts et structures de croyances qui influencent la vie humaine dans les sociétés occidentales modernes. Il paraît probable, par exemple, que le concept scientifique populaire de "gène", soit intimement lié aux conceptions humaines sur les agents. Suite aux écrits de théoriciens tel Richard Dawkins et à ceux du journalisme scientifique populaire, d'une part, plusieurs personnes croient que les gènes sont des agents internes cachés qui ont leurs propres programmes et qui influencent nos motivations et sentiments. D'autre part, certains scientifiques ont des conceptions plus sophistiquées et plus nuancées des gènes et de leur rapport avec les processus cellulaires et évolutifs. Cette analogie suggère qu'il puisse y avoir, entre les structures de croyances religieuses populaires et la connaissance contemplative développée dans certaines communautés religieuses, une relation du même type que celle qu'on identifierait, dans les sociétés occidentales modernes, entre les structures des croyances scientifiques populaires et la connaissance scientifique. 
Même si j'ai attiré l'attention sur les différences entre mon projet et celui de Boyer, celui-ci avance tout de même une affirmation qui pourrait être interprétée comme une remise en question de mon approche. Il soutient qu'il n'y a pas «d'instinct de transcendance» chez les êtres humains et ainsi que la religion ne peut être comprise (du moins du point de vue de la psychologique évolutive) en faisant appel à la transcendance. Mon objection à cette affirmation est qu'elle présuppose la notion problématique d' «instinct mental». Il est impossible, je crois, d'invoquer le concept d'instinct sans s'embourber dans la dichotomie entre le naturel et le culturel, entre l'inné et l'acquis, l'instinctif et l'appris. Je suis d'accord avec ces théoriciens de la biologie et de la psychologie qui soutiennent que nous avons besoin de remplacer ce cadre dichotomique par une approche axée sur les "systèmes développementaux» (voir Oyama 2002; Oyama et al. 2001). Selon la théorie des systèmes développementaux, «l'inné» (ou l'instinctif) et «l'acquis » ne désignent pas deux classes de caractéristiques développementales mutuellement exclusives. D'une part, les traits phénotypiques sont «acquis » autant qu' «innés » parce qu'ils doivent être construits développementalement, c'est-à-dire acquis par ontogénie. D'autre part, les conditions environnementales sont autant «innées » qu' "acquises » parce qu'elles sont inséparablement transmises avec les gènes et entrent ainsi dans la formation de l'organisme dès le départ. Comme Susan Oyama le soutient éloquemment dans son livre The Ontogeny of Information, la question «n'est pas que les gènes et l'environnement s'avèrent nécessaires pour toutes les caractéristiques, innées ou acquises (le point de vue habituel de gens bien informés), mais qu'il n'existe pas de distinction intelligible entre les caractéristiques de l'inné (celles qui ont une base biologique ou génétique) et celles de l'acquis (par la médiation environnementale)» (2002, 138). C'est pourquoi je suis sceptique devant tout modèle d'explication qui tente de mettre à part une catégorie de capacités biologiques et mentales en les étiquetant comme étant des «instincts".

Quel est le rapport avec la religion? Boyer croit que nous avons certains instincts qui s'expriment dans nos présupposés intuitifs sur les agents et les relations sociales, et que ces instincts structurent des concepts religieux, tel celui d'agent surnaturel. Par ailleurs, d'autres tendances religieuses, croit-il, ne sont pas fondées sur l'instinct. En s'appuyant là-dessus, il déclare qu'il n'y a pas d'instinct de transcendance chez les 
êtres humains et que, par conséquent, la religion ne peut pas être comprise en se fondant sur la transcendance.

Ma réponse à cela est que cette notion d' «instinct» n'est pas d'un grand secours. Il n'y a pas d'instincts, parce que le terme n'a pas d'application claire. Les cycles de vie organismiques se propagent d'une génération à l'autre en se reconstruisant dans un processus de développement, plutôt qu'en se déployant selon des plans ou des programmes transmis génétiquement. Les processus de reconstruction développementale impliquent de nombreux éléments causaux indépendants, qui sont liés entre eux par un rapport de réciprocité, en tant que processus et produits, au lieu d'appartenir aux catégories, conceptuellement dichotomisées, de nature génétique versus culture environnementale. Il n'existe donc aucune assise scientifique solide pour essayer de comprendre les normes et les concepts religieux en utilisant le construit explicatif d'«instincts». C'est pourquoi je récuse l'affirmation qu'il n'existe pas d'instinct humain de transcendance - non pas parce que je crois qu'il existe un tel instinct, mais parce que le concept d' "instinct» est tout simplement inapplicable au développement biologique et culturel.

Ce débat en psychologie et en biologie à propos du concept d'instinct a une portée significative par rapport à notre question de départ. Une fois que l'on a écarté le concept d'instinct, on est libre de dire que certaines normes et certains concepts religieux, et assurément certaines expériences religieuses - en particulier celles qu'on trouve dans des traditions contemplatives bien développées - peuvent très bien devoir être expliqués comme le produit d'une aspiration humaine à la transcendance, une aspiration qui peut être maintenue et transmise culturellement de génération en génération. La psychologue développementaliste Margaret Donaldson, par exemple, a établi la séquence de ce genre d'aspiration en la mettant en corrélation avec des modes de développement intellectuel humain et émotionnel au cours de la vie humaine; cela se manifeste notamment dans ce qu'elle appelle les modes d'expérience de la transcendance axés sur la perception des valeurs, cultivées par les traditions contemplatives du monde (voir Donaldson 1991). Du point de vue des systèmes développementaux, qui rejettent le concept d'instinct, il n'y a pas d'empêchement théorique à reconnaître que l'aspiration humaine à des modes transcendants d'expérience contemplative puisse faire partie des ressources développementales qui structurent l'esprit humain dans certaines sociétés et traditions. 
Une caractéristique commune aux trois manières d'aborder la science et la religion que j'ai opposées à mon approche de circulation mutuelle est qu'elles tiennent les concepts de «science » et de « religion » largement pour acquis. Ces concepts, cependant, sont très problématiques. Ce sont des catégories intellectuelles européennes, élaborées dans un contexte d'affrontements entre la science et la religion, à une époque récente dans l'histoire de l'Occident, celle des Lumières et de la modernité. Elles ne fournissent aucune indication précise sur les processus de formation du savoir ou sur les pratiques sociales de diverses autres traditions culturelles, en particulier celles des traditions de contemplation ou de sagesse asiatiques (voir Hut 2003). Comme Wallace l'a récemment écrit dans son introduction à un volume sur le bouddhisme et la science:

L'assertion que le bouddhisme comporte des éléments scientifiques n'occulte en rien les composantes explicitement religieuses de cette tradition [...]. Le bouddhisme se préoccupe principalement des buts de l'être humain, de son sens et de sa valeur. Mais comme la science, il se préoccupe aussi de comprendre le domaine de l'expérience sensorielle et mentale, et il pose les questions de la composition et du fonctionnement de l'univers, dans ses phénomènes objectifs aussi bien que subjectifs [...]. Le bouddhisme aborde les questions portant sur le sens de la vie, nos origines et notre destinée ultime, et les expériences de notre vie intérieure. Mais le simple fait que le bouddhisme comporte des éléments de la religion ne suffit pas pour le catégoriser spécifiquement comme religion, tout comme il n'est pas possible de le classer totalement comme science. Pour être en mesure d'étudier cette discipline objectivement, nous devons nous départir de nos catégories conceptuelles habituelles et nous préparer à être confrontés par quelque chose qui nous est complètement étranger et qui peut remettre en question nos présupposés les plus profonds. Il se peut qu'au cours de l'opération, nous en venions à réévaluer le statut de la science elle-même, par rapport aux axiomes métaphysiques sur lesquels ce statut est fondé. (2003, 9-10)

Dans cet essai (et dans Verala, Thompson et Rosch 1991), j'ai soutenu que certaines traditions de contemplation ou de sagesse (surtout, mais pas seulement, le bouddhisme) et certaines manières d'aborder la science (l'approche dite "d'inscription corporelle» dans la science cognitive et son élaboration plus récente dans le programme de recherche de «neurophénoménologie» - voir Lutz et Thompson 2003) ne sont pas simplement compatibles, mais s'enrichissent et s'éclairent mutuellement. 
Grâce à des mouvements d'aller et retour, chacune de ces approches peut contribuer à restructurer l'autre, et il en résulte de nouvelles compréhensions conceptuelles et pratiques pour les deux.

Ce qui est en jeu, ultimement, dans ces développements n'est pas simplement de savoir si nous pouvons nous doter d'une science de l'esprit humain qui atteigne une certaine maturité au plan de sa méthode; il s'agit plutôt de savoir si nous pouvons nous doter d'une science de l'esprit qui soit mature sur le plan éthique et informée sur le plan spirituel. Autrement dit, permettre à la subjectivité et à l'expérience contemplative de jouer un rôle actif et créatif dans le domaine de la science cognitive relève aussi bien de l'éthique que de la méthodologie. Mon souhait, à long terme, serait de voir prospérer de mon vivant une science de l'esprit contemplative, phénoménologique et expérimentale.

\section{Références}

Boyer, P. (2001), Religion Explained: The Evolutionary Origins of Religious Thought, New York, Basic Books.

- (2005), "Gods, Spirits, and the Mental Instincts that Create Them », dans J. Proctor, dir., Science, Religion, and the Human Experience, New York, Oxford University Press, p. 237-260.

Buccino, G., F. Binkofski, G.R. Fink, L. Fadiga, L. Fogassi, V. Gallese, R.J. Seitz, K. Rizzolatti et H.J. Freund (2001), «Action Observation Activates Premotor and Parietal Areas in a Somatotopic Manner: An fMRI Study ", European Journal of Neuroscience, 13, p. 400-404.

Depraz, N. (2001), "The Husserlian Theory of Intersubjectivity as Alterology:

Emergent Theories and Wisdom Traditions in the Light of Genetic Phenomenology ", Journal of Consciousness Studies, 8/5-7, p. 169-178. Repris dans E. Thompson, dir., Between Ourselves: Second Person Issues in the Study of Consciousness, Thorverton, Imprint Academic, p. 169-178.

Depraz, N., F.J. Varela et P. Vermersch (1999), "The Gesture of Awareness: An Account of Its Structural Dynamics ", dans M. Velmans, dir., Phenomenal Consciousness, Amsterdam - Philadelphia, John Benjamins, p. 121-136.

Depraz, N., P. Vermersch et F.J. Varela (2003), On Becoming Aware: A Pragmatics of Experiencing, Amsterdam - Philadelphia, John Benjamins.

De WaAl, F.B.M. (1996), Good Natured: The Origins of Right and Wrong in Humans and Other Animals, Cambridge, Harvard University Press. 
_- (2002), "On the Possibility of Animal Empathy", dans T. Manstead, N. Fridja et A. FIscher, dir., Feelings and Emotions: The Amsterdam Symposium, Cambridge, Cambridge University Press, p. 381-401 (consulté sous une forme dactylographiée).

Donaldon, M. (1991), Human Minds: An Exploration, London, Penguin Books.

Dreyfus, G. (2002), «Is Compassion an Emotion? A Cross-Cultural Exploration of Mental Typologies ", dans R. J. Davidson et A. Harrington, dir., Visions of Compassion: Western Scientists and Tibetan Buddhists Examine Human Nature, New York, Oxford University Press, p. 31-45.

Gallagher, S. (1986), "Body Image and Body Schema: A Conceptual Clarification », The Journal of Mind and Behavior, 7, p. 541-554.

- (2001), "The Practice of Mind: Theory, Simulation, or Primary Interaction?", Journal of Consciousness Studies, 8/5-7, p. 83-108. Repris dans E. Thompson, dir., Between Ourselves: Second Person Issues in the Study of Consciousness, Thorverton, Imprint Academic, p. 83-108.

Gallup Jr., G.G. (1998), "Can Animals Empathize? Yes », Scientific American, 9, p. 65-75.

Gould, S.J. (1999), Rocks of Ages. Science and Religion in the Fullness of Life, New York, Ballantine.

Gupta, B. (1998), The Disinterested Witness: A Fragment of Advaita Vedanta Phenomenology, Evanston, Northwestern University Press.

Husserl, E. (2001), Analyses Concerning Passive and Active Synthesis. Lectures on Transcendental Logic / trad. par A.J. Steinbock, Dordrecht, Kluwer Academic Publishers.

Hut, P. (2003), "Conclusion: Life as a Laboratory", dans B.A. Wallace, dir., Buddhism and Science: Breaking New Ground, New York, Columbia University Press, p. 399-416.

Johnson, M. (1993), Moral Imagination: Implications of Cognitive Science for Ethics, Chicago, University of Chicago Press.

Levenson, R.W. et A.M. Reuf (1992), «Empathy: A Physiological Substrate », Journal of Personality and Social Psychology, 63, p. 234-246.

Lutz, A. et E. Thомpson (2003), « Neurophenomenology. Integrating Subjective Experience and Brain Dynamics in the Neuroscience of Consciousness ", Journal of Consciousness Studies, 10, p. 31-52. 
Lutz, A., J.P. Lachaux, J. Martinerie et F.J. Varela (2002), "Guiding the Study of Brain Dynamics by Using First-Person Data: Synchrony Patterns Correlate with Ongoing Conscious States During a Simple Visual Task», Proceedings of the National Academy of Sciences USA, 99, p. 1586-1591.

Marbach, E. (1998), "How to Study Consciousness Phenomenologically or Quite a Lot Comes to Mind", Journal of the British Society for Phenomenology, 19/3, p. 252-268.

McGee, M. (2000), Transformations of Mind: Philosophy as Spiritual Practice, Cambridge, Cambridge University Press.

McGeer, V. (2001), "Psycho-practice, Psycho-theory and the Contrastive Case of Autism ", Journal of Consciousness Studies, 8/5-7, p. 109-132. Repris dans E. Thompson, dir., Between Ourselves: Second Person Issues in the Study of Consciousness, Thorverton, Imprint Academic, p. 109-132.

Newberg, A., E. D’Aquili et V. Rause (2001), Why God Won't Go Away: Brain Science and the Biology of Belief, New York, Ballantine Books.

Oyama, S. $\left(2002^{2}, 1985\right)$, The Ontogeny of Information: Developmental Systems and Evolution, Durham, Duke University Press.

Oyama, S., P.E. Griffiths et R.D. Gray, dir. (2001), Cycles of Contingency: Developmental Systems and Evolution, Cambridge, The MIT Press.

Pitkin, A. (2001), "Scandalous Ethics: Infinite Presence with Suffering », Journal of Consciousness Studies, 8/5-7, p. 232-246. Repris dans E. Thompson, dir., Between Ourselves: Second Person Issues in the Study of Consciousness, Thorverton, Imprint Academic, p. 232-246.

Povinelui, D.J. (1998), "Can Animals Empathize? Maybe Not», Scientific American, 9, p. 65-75.

Preston, S. et F.B.M. De WaAl (2002), «Empathy: Its Ultimate and Proximate Bases ", Behavioral and Brain Sciences, 25, p. 1-72.

Proctor, J. dir., (2005), Science, Religion, and the Human Experience, New York, Oxford University Press.

Shantideva (1997), The Way of the Bodhisattva / trad. par The Padmakara Translation Group, Boston, Shambala Press (= 1992 La marche vers l'éveil, Paris, Padmakara).

Stein, E. (1964) [allemand 1917], On the Problem of Empathy / trad. par W. Stein, La Hague, Martinus Nijhoff.

TaYlor, E. (1996), William James, on Consciousness beyond the Margin, Princeton, Princeton University Press. 
Thompson, E. et F.J. Varela (2001), «Radical Embodiment: Neural Dynamics and Consciousness", Trends in Cognitive Sciences, 5, p. 418-425.

Tomasello, M. (1999), The Cultural Origins of Human Cognition, Cambridge, Harvard University Press.

Varela, F.J., E. Thompson et E. Rosch (1991), The Embodied Mind: Cognitive Science and Human Experience, Cambridge, MIT Press.

Varela, F.J. et J. Shear, dir. (1999), The View from Within: First-Person Approaches to the Study of Consciousness, Thorverton, Imprint Academic.

Vetlesen, A.J. (1994), Perception, Empathy, and Judgment: An Inquiry into the Preconditions of Moral Performance, University Park, Pennsylvania State University Press.

Wallace, B.A. (2003), "Introduction: Buddhism and Science», dans B.A. WALLACE, dir., Buddhism and Science: Breaking New Ground, New York, Columbia University Press, p. 9-10.

- (2005), "The Intersubjective Worlds of Science and Religion", dans J. Proctor, dir., Science, Religion, and the Human Experience, New York, Oxford University Press, p. 309-328.

Zahavi, D. (1999), Self-Awareness and Alterity: A Phenomenological Investigation, Evanston, Northwestern University Press.

(2001), «Beyond Empathy: Phenomenological Approaches to Intersubjectivity ", Journal of Consciousness Studies, 8/5-7, p. 151-167. Repris dans E. Thompson, dir., Between Ourselves: Second Person Issues in the Study of Consciousness, Thorverton, Imprint Academic, p. 151-167.

\section{Résumé}

Cet article pose la question à savoir comment la science et la religion peuvent émerger de l'expérience humaine, tout en transcendant cette dernière. Un des moyens de répondre à la question consiste à se demander quelle forme doit prendre une science mature de l'esprit humain. L'auteur estime qu'une telle science doit articuler des méthodes de première personne de l'expérience subjective avec des méthodes de troisième personne des sciences biobehavioristes. L'auteur entend démontrer la fécondité d'une approche dite de «circulation mutuelle» entre la science cognitive, la phénoménologie et la psychologie contemplative pour en arriver à une telle science mature, approche qui dépasse le traditionnel binôme science-religion. Il exemplifie cette approche en l'appliquant à l'expérience de l'empathie, cas type qui permet de faire ressortir l'intersubjectivité de l'expérience humaine. 


\begin{abstract}
This article addresses the question of how science and religion emerge from yet transcend human experience. An important pointer to this interrogation consists in the form a mature science of the mind should take. The author suggests that such a science should articulate together first person methods of subjective experience with third person methods of biobehavioral sciences. In order to do so he puts forward a "mutual circulation" approach that combines cognitive science, phenomenology and contemplative psychology. This approach has the merit of dismantling the traditional science-religion pair. He exemplifies this approach by applying it to an experience which emphasizes the intersubjectivity of human experience, empathy.
\end{abstract}

(C) Revue Théologiques 2004. Tout droit réservé. 